Research Article

\title{
An Integrated Production-Distribution Planning Problem under Demand and Production Capacity Uncertainties: New Formulation and Case Study
}

\author{
Taycir Ben Abid (D, Omar Ayadi, and Faouzi Masmoudi \\ Mechanics, Modelling and Production Research Laboratory (LA2MP), University of Sfax, \\ National Engineering School of Sfax (ENIS), Road Soukra B.P.1173-3038, Sfax, Tunisia \\ Correspondence should be addressed to Taycir Ben Abid; taicir.benabid@enis.tn
}

Received 27 December 2019; Accepted 18 February 2020; Published 13 April 2020

Academic Editor: Anna M. Gil-Lafuente

Copyright (C) 2020 Taycir Ben Abid et al. This is an open access article distributed under the Creative Commons Attribution License, which permits unrestricted use, distribution, and reproduction in any medium, provided the original work is properly cited.

In this study, we propose to solve a biobjective tactical integrated production-distribution planning problem for a multisite, multiperiod, multiproduct, sea-air intermodal supply chain network under uncertainties. Two random parameters are considered simultaneously: product replenishment orders and production capacity, which are modelled via a finite set of scenarios, using a two-stage stochastic approach. A corresponding mathematical model is developed, coded, and solved using the LINGO 18.0 software optimisation tool. This model aims to simultaneously minimise the total costs of production in both regular and overtime, inventory, distribution, and backordering activities and maximise the customer satisfaction level over the tactical planning horizon. The AUGMECON technique is applied to handle with the multiobjective optimisation. The applicability and the performance of the proposed model are tested through a real-life case study inspired from a medium-sized Tunisian textile and apparel company. Sensitivity analysis on stochastic parameters and managerial insights for the studied supply chain network are argued based on the empirical findings.

\section{Introduction}

The tremendous competition in productivity, quality and time response, introduction of products with short life cycles, and intensive customers' expectations are the main concerns of today's global markets. These circumstances have pushed industries to aggressively seeking better ways to establish a closer interaction and collaboration between the different activities of the supply chain [1]. Handling decisions of different functions of the supply chain such as production planning and distribution planning are vital for companies to obtain an optimal strategy that improves efficiency and enhances the global performance. Due to the complex nature and dynamics of the relationships amongst the different actors and the hidden customer preference, firms have faced the threat of many sources of uncertainties such as customer demand, prices, and resource capacities
[2]. Underestimating uncertainties and failing to account for their significant fluctuations in supply chain planning could lead to unsatisfied customer demand translating to loss of market share. It ultimately leads to undesirable and unexpected impacts on the ability of reaching supply chain goals. Thus, a major challenge for the decision-maker is to determine how to continuously manage and monitor these uncertainties.

In this context, we propose to develop a biobjective integrated production-distribution planning model under both demand and production capacity uncertainties. A twostage stochastic programming formulation is proposed to help the decision-makers to simultaneously minimise total costs and maximise the customer satisfaction level in terms of on-time delivery. A case study of a manufacturing company producing and exporting fashion products to the European market is tackled in this paper. The efficiency of 
the proposed approach is illustrated throughout a comparison with the current planning of the company.

The remaining of this paper is structured as follows: A literature review of related works is highlighted in Section 2. Section 3 is devoted to the problem statement considering a two-stage stochastic model for an integrated productiondistribution planning problem under uncertainties. The proposed mathematical model is developed in Section 4. The resolution method of the multiobjective optimisation is described in Section 5. In Section 6, a computational study inspired from the textile and apparel industry is presented to assess the performance of the proposed two-stage stochastic programming method. Section 7 displays the related results as well as sensitivity analysis on the variability of stochastic parameters and the main managerial insights. Finally, conclusions and future works are highlighted in Section 8.

\section{Related Literature}

The coordinated production and distribution planning problems have drawn more and more attention for many years. As these problems are mutually related, it is critical to optimise these two functions and plan them simultaneously in an integrated manner instead of optimising in a sequential way with little or no integration [3]. There are a number of review papers on supply chain management tackling this issue $[4,5]$. Traditionally, the field of supply chain design has focused on mono-objective optimisation quantifying economic objectives, such as cost minimisation [6, 7] and profit maximisation $[8,9]$. Nevertheless, real-world problems often require the consideration of multiple conflicting criteria to measure the customer service level and supply chain responsiveness. The multiobjective optimisation mainly includes goals related to maximising products' quality level [10], minimising the average tardiness, and minimising delivery [11]. Owing to the difficulties of collecting data and knowledge about production and distribution planning problems, some works examined real-life case studies such as automotive manufacturing [11], apparel industry [12], pharmaceutical industry [13], and plastic industry [14]. Many researches suppose the availability of deterministic data when developing a production-distribution planning model. However, the real-world planning problems imply some level of uncertain and incomplete data in one or more of the input parameters. The literature reveals that optimisation problems with uncertainty have been widely tackled and developed. Many survey articles on such models have appeared in $[1,15,16]$. There are diverse factors that generate uncertainties in supply chains. The most commonly and extensively stated sources of uncertainty are connected to the customers' demand, process, and supply that have huge effects on the decisionmaking process. To incorporate uncertainty in an optimisation model, several approaches can be addressed such as stochastic programming methods [17], fuzzy programming [10], and robust optimisation techniques [18]. One of the most widely used formulations for decisionmaking under uncertainty is stochastic programming with recourse. The basic idea of this modelling approach is to formulate the problem in a two-stage setting. This formulation was introduced for the first time in [19]. Another main issue in scenario-based (two- and multistage) stochastic programs consists of generating scenarios associated with their probabilities. Some studies of the production-distribution modelling efforts in the literature that are more relevant to our paper are summarised in Table 1.

In the context of stochastic optimisation, many authors state that the most obvious and extensively studied source of uncertainty has been demand. For instance, Guillén et al. [25] investigated a two-stage stochastic model to incorporate the uncertainty associated with the demand and aimed for the maximisation of profit, demand satisfaction, and financial risk. In addition to the demand uncertainty, several other uncertain parameters have been considered in SC network designs including prices, cost, capacities, and lead time. Rahimi et al. [27] used two-stage stochastic programming considering demand and cost uncertainties. Mohammadi and Mohd [23] proposed a two-stage stochastic programme under uncertainties of costs, demand, and capacity of facilities. Azaron et al. [26] focused on a multiobjective two-stage stochastic programming approach for the supply chain network design in which demands, supplies, processing time, shortage, transportation, and capacity costs were assumed as uncertain parameters. Mirzapour Al-e-hashem et al. [24] addressed a multisite, multiperiod, multiproduct three-echelon supply chain under uncertainties of cost parameters and demand fluctuations. Nemati and Alavidoost [29] studied a multiobjective model through the integration of sales, production, distribution, and procurement planning considering sea-air intermodal transportation modes. The aims were to minimise the total supply chain cost and to minimise the customer service level in terms of maximising total sales. Pasandideh et al. [28] proposed a biobjective optimisation of the supply chain network under uncertainties of costs, customer demand, unit processing time, available production time, and time of setup.

To the best of our knowledge, we notice that it is the first time that the uncertainty of the production capacity in terms of workforce availability is considered in a two-stage stochastic programming approach to model a biobjective tactical integrated production-distribution planning considering sea-air intermodal transportation. Finally, thanks to a real-world case study of the textile-apparel industry, we contemplate a more realistic situation than those examined in $[20,21]$ by considering two sources of uncertainty and multiobjective scheme in our production-distribution model in order to minimise total costs along with maximising the customer satisfaction level in terms of minimising late deliveries.

\section{Problem Statement}

The supply chain network, illustrated in Figure 1, consists of multiple production stages composed of several production plants that receive and transform raw materials to 


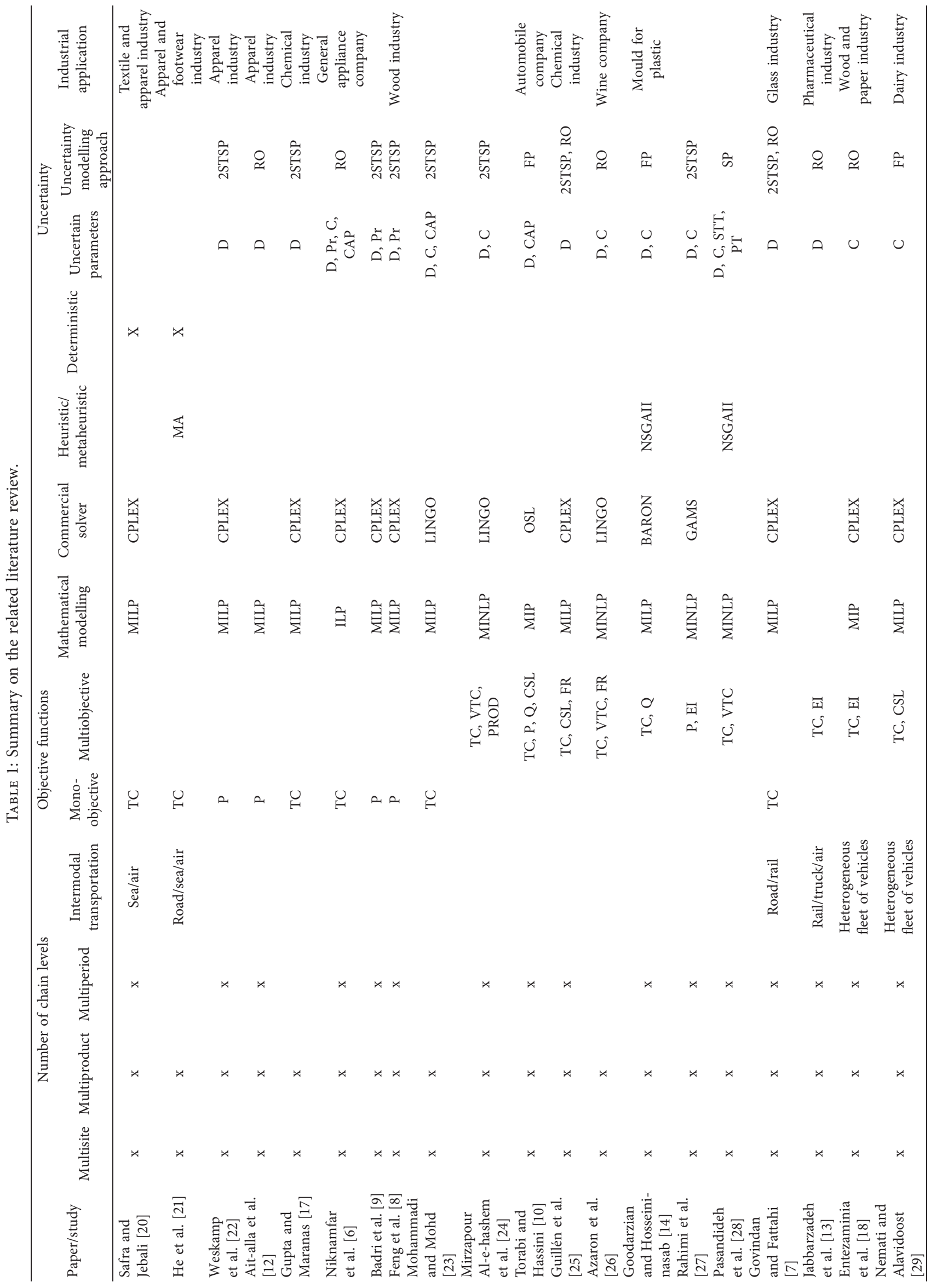




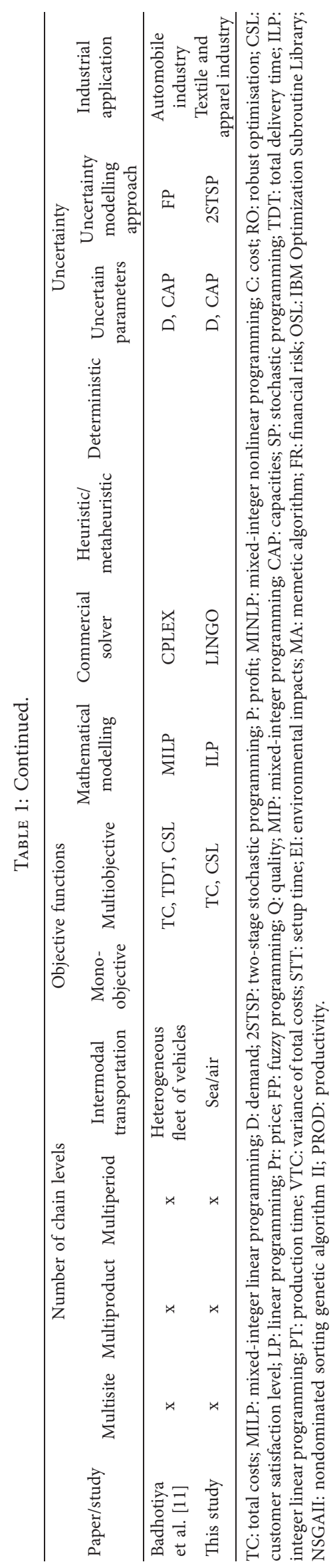




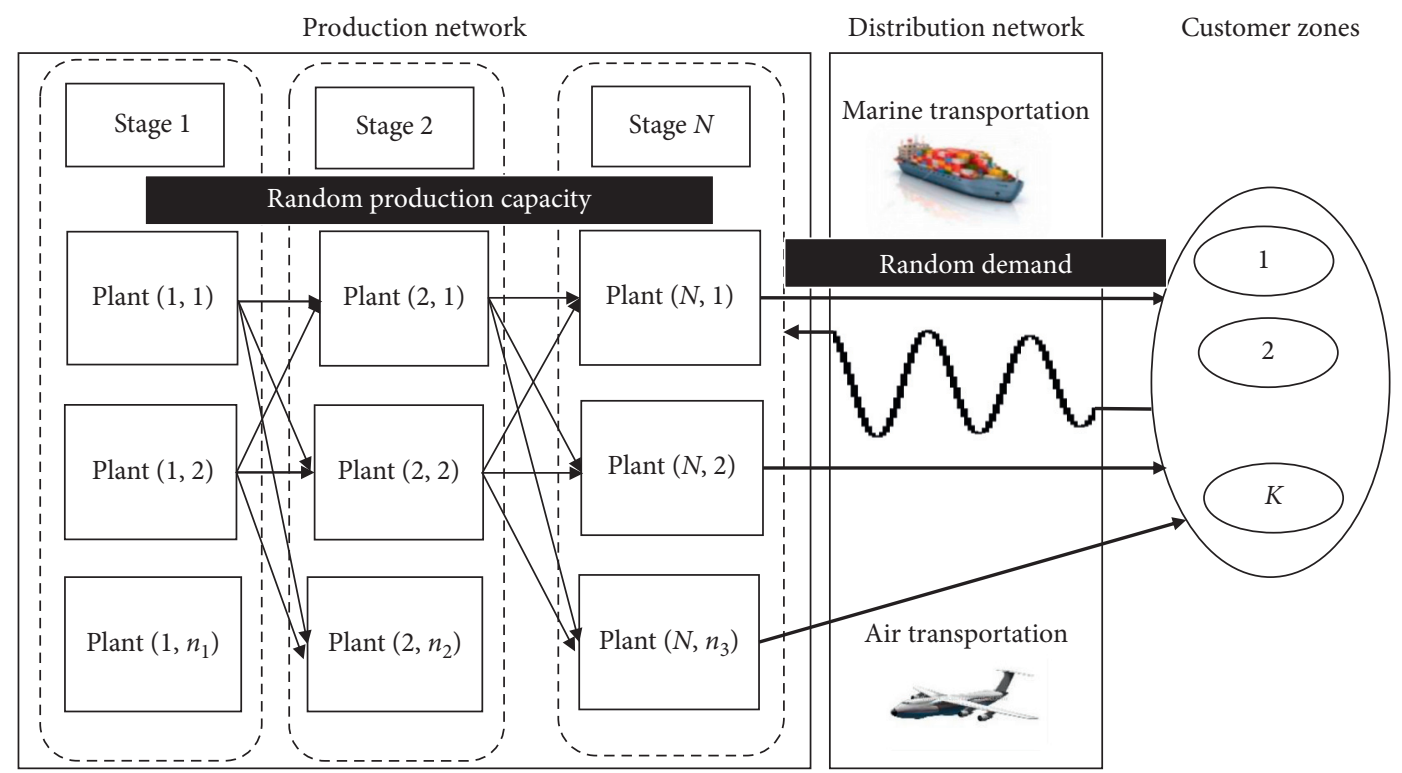

FIGURE 1: Supply chain network for the integrated production-distribution planning problem.

semifinished and end products and deliver them to the customer zones.

The tactical planning horizon is often divided into several periods. Note that each plant is characterized by its own production processing time, costs, and capacities. The transportation time between factories from the last production stage and customers' zones depends on the used transportation modes: marine or air transportation. The shortage of not meeting customers' demand on time is acceptable with penalty cost. Nevertheless, all the orders should be fully met at the end of the tactical planning horizon since lost sales are not permitted. Both stochastic demand and stochastic production capacity are considered in this problem, and we assume that these uncertainties can be modelled by defining several different scenarios with a given probability of occurrence. We assume that the company has two types of customers' orders: firm orders that are supposed to be known with certainty at the beginning of the planning horizon and replenishment orders with short due dates that can be processed from a given period. The latter is a part of demand that cannot be initially predicted. So, it will be considered a stochastic order. Moreover, there are a variety of internal factors, which cannot be easily identified and cause huge variations in workers' productivity. We note that the most important ones are the resource availability, workforce skills, and breakdown machines. In our study, we would focus on the workforce absenteeism factor, which leads to a significant fluctuation of the production capacity.

For the considered tactical planning horizon, the optimisation goals are firstly minimising the overall costs, which mainly include production costs in normal working hours and in overtime, inventory-holding costs, shipping costs between production plants, distribution costs to the final customers, and backorder costs. Secondly, we aim to maximise the customer service level by ensuring the delivery of demand on time.
We develop a two-stage scenario-based stochastic programming planning problem, which is formally defined in terms of the following assumptions:

(i) Customer demand and production capacity are considered random parameters.

(ii) The initial values of inventory and backlog are null.

(iii) Production plants from the last stage deliver products directly to overseas customer zones bypassing the distribution network (airline and waterway modes).

(iv) It is assumed that all the products produced and transported have no defects.

(v) Backorder and overtime production decisions are allowed to avoid volatility in demand and production capacity.

(vi) Demand needs not be fully fulfilled all the time since backorders are allowed. However, the lost sales are not permitted.

\section{Mathematical Formulation}

This section describes the two-stage stochastic programming approach for an integrated production-distribution planning problem and formulates the mathematical model. We examine a biobjective stochastic integrated productiondistribution planning problem using sea-air intermodal transportation.

The stochastic two-stage programming approach represents the random event through a finite number $S=\left\{\gamma_{1}, \gamma_{2}, \ldots, \gamma_{s}\right\}$ of possible realisations, called scenarios with known probabilities $\delta(s)$, and basically, the decisions are made in two different stages. In this approach, first-stage decisions are made "here and now" at the beginning of the planning horizon, prior to the realisation of the random events. After the first-stage decisions are taken and the 
random events are realised, the second-stage or recourse decisions always referred to as "wait-and-see" decisions are taken as a corrective action after the realisation of the uncertainty [30]. The presence of uncertainty is correlated with the stochastic nature of the demand and production capacity associated with the second-stage decisions. Therefore, the objective function consists of the sum of the first-stage decision costs and the expected second-stage recourse costs.

To formulate the problem mathematically, the sets, indices, both deterministic and random parameters, and decision variables are introduced in Table 2 . Let ST, $I, P, M$, and $K$ be the sets of production stages, products, plants, transportation modes, and customers, respectively. The representation set for the probability and the number of scenarios are expressed as $S$ and $\delta(s)$, respectively (Table 2).

Based on the abovementioned assumptions and description, the two-stage stochastic programming model for the tactical production and distribution planning problem could be formulated as a multiobjective integer linear programming model. The objectives correspond to minimisation of the overall costs of the supply chain and maximisation of customer services that can be rendered to customers in terms of the rate of on-time deliveries.

4.1. Objective Functions

$$
\begin{aligned}
\operatorname{MIN}\left(F_{1}\right)= & \sum_{s=1}^{S} \delta(s) *\left(\sum_{t=t_{1}}^{T} \sum_{p=1}^{P} \sum_{i^{\prime}, i=1}^{I} \mathrm{CI}_{i p}\left(I_{i p t, s}+\mathrm{SF}_{i p t, s}\right)+\mathrm{CP}_{i p} P_{i p t, s}+\mathrm{CO}_{i p} O_{i p t, s}+\mathrm{CSH}_{i i^{\prime} p} \mathrm{SH}_{i i^{\prime} p t, s}\right. \\
& \left.+\sum_{t=1}^{T} \sum_{k=1}^{K} \sum_{p=1}^{P} \mathrm{CB}_{p k} B_{p k t, s}+\sum_{t=1}^{T} \sum_{p=1}^{P} \sum_{i \in S T_{N}} \sum_{k=1}^{K} \sum_{m=1}^{2} \mathrm{CD}_{i p k m} \mathrm{OQ}_{i p k m t, s}\right) \\
& +\sum_{t=1}^{t_{1}} \sum_{p=1}^{P} \sum_{i^{\prime}, i=1}^{I} \mathrm{CP}_{i p} P_{i p t}+\mathrm{CO}_{i p} \mathrm{O}_{i p t}+\mathrm{CSH}_{i i^{\prime} p} \mathrm{SH}_{i i^{\prime} p t}+\mathrm{CI}_{i p}\left(I_{i p t}+\mathrm{SF}_{i p t}\right) \\
\operatorname{MAX}\left(F_{2}\right)= & \sum_{s=1}^{S} \delta(s) * \frac{\sum_{t=1}^{T} \sum_{p=1}^{P} \sum_{k=1}^{K}\left(\mathrm{RO}_{p k t, s}+D_{p k t}-B_{p k t, s}\right)}{\sum_{t=1}^{T} \sum_{p=1}^{P} \sum_{k=1}^{K} \mathrm{RO}_{p k t, s}+D_{p k t}}
\end{aligned}
$$

\subsection{Constraints}

$$
\begin{aligned}
I_{i p t} & =I_{i p t-1}+P_{i p t}+O_{i p t}-\sum_{i^{\prime} \in \mathrm{SUCC}_{i}} \mathrm{SH}_{i i^{\prime} p t}, \quad \forall p, t \leq t_{1}, i \in \mathrm{ST}_{j \leq N-1}, \\
I_{i p t, s} & =I_{i p t-1, s}+P_{i p t, s}+O_{i p t, s}-\sum_{i^{\prime} \in \mathrm{SUCC}_{i}} \mathrm{SH}_{i i^{\prime} p t, s}, \quad \forall s, p, t \geq t_{1}, i \in \mathrm{ST}_{j \leq N-1}, \\
I_{i p t, s} & =I_{i p t-1, s}+P_{i p t, s}+O_{i p t, s}-\sum_{i \in \mathrm{ST}_{N}} \sum_{k=1}^{K} \sum_{m=1}^{2} \mathrm{OQ}_{i p k m t, s}, \quad \forall p, t \geq t_{1}, s, i \in \mathrm{ST}_{N}, \\
I_{i p t} & =I_{i p t-1}+P_{i p t}+\mathrm{O}_{i p t}-\sum_{i \in \mathrm{ST}_{N}} \sum_{k=1}^{K} \sum_{m=1}^{2} \mathrm{OQ}_{i p k m t, s}, \quad \forall p, t<t_{1}, s, i \in \mathrm{ST}_{N}, \\
\mathrm{SF}_{i p t, s} & =\mathrm{SF}_{i p t-1, s}-P_{i p t, s}-O_{i p t, s}+R_{i p t, s}, \quad \forall p, t \geq t_{1}, i, s, \\
\mathrm{SF}_{i p t} & =\mathrm{SF}_{i p t-1}-P_{i p t}-O_{i p t}+R_{i p t}, \quad \forall p, t<t_{1}, i, \\
B_{p k t, s} & =D_{p k t}+\mathrm{RO}_{p k t, s}+B_{p k t-1, s}-\sum_{i \in S_{N}} \sum_{m=1}^{2} \mathrm{OQ}_{i p k m}\left(t-\mathrm{DLT}_{m}\right), s^{\prime}, \quad \forall p, k, s, i \in \mathrm{ST}_{N}, t \geq \mathrm{DLT}_{m},
\end{aligned}
$$


TABLE 2: List of notations: sets, parameters, and decision variables.

\begin{tabular}{|c|c|c|}
\hline & Notations & Definitions \\
\hline Sets & $\begin{array}{l}\mathrm{ST}_{j} \\
I \\
\mathrm{SUCC}_{i} \\
P \\
T \\
M \\
S\end{array}$ & $\begin{array}{c}\text { Set of stages of production } j \in\{1,2, \ldots, N\} \\
\text { Set of plants indexed by } i \in\{1,2, \ldots, n\} \\
\text { Set of production plants that are direct successors of the plant } i, i \in\{2, \ldots, n\} \\
\text { Set of products indexed by } p €\{1,2, \ldots, P\} \\
\text { Set of periods indexed by } t \in\{1,2, \ldots, T\} \\
\text { Set of transportation modes } m \in M=\{m=1: \text { ship, } m=2 \text { : airplane }\} \\
\text { Scenario set } s \in\{1,2, \ldots, S\}\end{array}$ \\
\hline Stochastic parameters & $\begin{array}{c}\delta(s) \\
\mathrm{RO}_{p k t, s} \\
\mathrm{CAPP}_{i t, s}\end{array}$ & $\begin{array}{l}\text { Occurrence probability of the scenario } s \text { where } \sum_{s=1}^{S} \delta(s)=1 \\
\text { Random replenishment order of the customer } k \text { for the product } p \text { in the period } t \text { over the scenario } s \\
\text { Uncertain regular production capacity of the plant } i \text { in the period } t \text { under the scenario } s\end{array}$ \\
\hline Deterministic parameters & $\begin{array}{l}D_{p k t} \\
\mathrm{CP}_{i p} \\
\mathrm{CO}_{i p} \\
\mathrm{CSH}_{i i^{\prime} p} \\
\mathrm{CD}_{i p k m} \\
\mathrm{CB}_{p k} \\
\mathrm{CI}_{i p} \\
\mathrm{CAPI}_{i t} \\
\mathrm{CAPD}_{m t} \\
\mathrm{CAPSH}_{i, i^{\prime} t} \\
\mathrm{CAPO}_{i t} \\
\beta \\
\operatorname{PLT}_{i p} \\
\mathrm{DLT}_{m}\end{array}$ & $\begin{array}{l}\text { Deterministic demand of the customer } k \text { for the product } p \text { in the period } t \\
\text { Unit regular production cost of the product } p \text { by the plant } i \\
\text { Unit overtime production cost of the product } p \text { by the plant } i \\
\text { Unit shipping cost of the product } p \text { from the plant } i \text { to the plant } i^{\prime}, i^{\prime} \in \text { SUCC }_{i} \\
\text { Unit distribution cost of the product } p \text { from the plant } i, i \in S_{j=N} \text {, to the customer } k \text { using the } \\
\text { Unit backorder cost of the product } p \text { in supplying the demand of the customer } k \\
\text { Unit inventory-holding cost of the product } p \text { in the plant } i \\
\text { Maximum storage capacity of the plant } i \text { in the period } t \\
\text { Distribution capacity of the transportation mode } m \text { in the period } t \\
\text { Shipping capacity between the plant } i \text { and its successor } i^{\prime} \text { in the period } t \\
\text { Overtime production capacity of the plant } i \text { in the period } t \\
\text { Maximum fraction of backorder variation allowed in the period } t, \beta \in[0 \ldots 1] \\
\text { Production lead time of the product } p \text { in the plant } i \\
\text { Distribution lead time using the transportation mode } m\end{array}$ \\
\hline $\begin{array}{l}\text { First-stage decision } \\
\text { variables }\end{array}$ & $\begin{array}{l}P_{i p t} \\
O_{i p t} \\
\mathrm{SH}_{i i^{\prime} p t} \\
R_{i p t} \\
I_{i p t} \\
\mathrm{SF}_{i p t}\end{array}$ & $\begin{array}{l}\text { Quantity of the product } p \text { produced by the plant } i \text { in regular time period } t \leq t_{1} \\
\text { Quantity of the product } p \text { produced by the plant } i \text { in overtime period } t \leq t_{1} \\
\text { Number of product units } p \text { shipped from the plant } i \text { to the plant } i^{\prime} \text { in the period } t \leq t_{1}, i^{\prime} \in \text { SUCC } \\
\text { Amount of products } p \text { received by the plant } i \text { in the period } t \leq t_{1} \\
\text { Inventory level of the end product } p \text { at the plant } i \text { at the end of the period } t \leq t_{1} \\
\text { Inventory level of the semifinished product } p \text { at the plant } i \text { at the end of the period } t \leq t_{1}\end{array}$ \\
\hline $\begin{array}{l}\text { Second-stage decision } \\
\text { variables }\end{array}$ & $\begin{array}{r}P_{i p t, s} \\
O_{i p t, s} \\
\mathrm{SH}_{i i^{\prime} p t, s} \\
R_{i p t, s} \\
I_{i p t, s} \\
\mathrm{SF}_{i p t, s} \\
\mathrm{OQ}_{i p k m t, s} \\
B_{p k t, s}\end{array}$ & $\begin{array}{l}\text { Quantity of the product } p \text { produced by the plant } i \text { in regular time period } t>t_{1} \text { under the scenario } s \\
\text { Quantity of the product } p \text { produced by the plant } i \text { in overtime period } t>t_{1} \\
\text { Number of product units } p \text { shipped from the plant } i \text { to the plant } i^{\prime} \text { in the period } t>t_{1} \text { under the } \\
\text { scenario } s, i^{\prime} \in \mathrm{SUCC}_{i} \\
\text { Amount of products } p \text { received by the plant } i \text { in the period } t>t_{1} \text { under the scenario } s \\
\text { Inventory level of the end product } p \text { at the plant } i \text { at the end of the period } t>t_{1} \text { under the scenario } \\
\text { Inventory level of the semifinished product } p \text { at the plant } i \text { at the end of the period } t>t_{1} \text { under the } \\
\text { scenario } s \\
\text { Amount of finished products } p \text { transported from } i \in \mathrm{ST}_{j=N} \text { to the customer } k \text { in the period } t \text { using } \\
\text { the transportation mode } m \text { under the scenario } s \\
\text { Backorder level of the product } p \text { incurred by the customer } k \text { at the end of the period } t \text { under the } \\
\text { scenario } s\end{array}$ \\
\hline
\end{tabular}

$$
\begin{aligned}
& B_{p k T, s}=0, \quad \forall p, k, s, \\
& R_{i^{\prime} p t+1}=\sum_{i^{\prime} \in \mathrm{SUCC}_{i}} \mathrm{SH}_{i i^{\prime} p t}, \quad \forall i, p, t, \\
& B_{p k t, s} \leq \beta\left(\mathrm{RO}_{p k t, s}+D_{p k t}\right), \quad \forall p, k, t, s, \\
& \sum_{p=1}^{P} \mathrm{PLT}_{i p} P_{i p t} \leq \mathrm{CAPP}_{i t, s}, \quad \forall i, t, s, \\
& \sum_{p=1}^{P} \mathrm{PLT}_{i p} \mathrm{O}_{i p t, s} \leq \mathrm{CAPO}_{i t}, \quad \forall i, t, s,
\end{aligned}
$$




$$
\begin{gathered}
\sum_{p=1}^{P} I_{i p t, s}+\mathrm{SF}_{i p t, s} \leq \mathrm{CAPI}_{i t}, \quad \forall i, t, s, \\
\sum_{p=1}^{P} \mathrm{SH}_{i i^{\prime} p t, s} \leq \mathrm{CAPSH}_{i i^{\prime} t}, \quad \forall i, i^{\prime} \in \mathrm{SUCC}_{i}, t, s, \\
\sum_{i \in S_{N}} \sum_{p=1}^{P} \sum_{k=1}^{K} \mathrm{OQ}_{i p k m t, s} \leq \mathrm{CAPD}_{m t}, \quad \forall m, k, t, s, \\
\left\{\mathrm{OQ}_{i p k m t, s}, B_{p k t, s}, \mathrm{SH}_{i i^{\prime} p t, s}, I_{i p t, s}, \mathrm{SF}_{i p t, s}, R_{i p t, s}, O_{i p t, s}, P_{i p t, s}\right\} \geq 0, \quad \forall i, p, t, k, m, s .
\end{gathered}
$$

The first objective function (1) aims to minimise the total expected costs of the whole supply chain including, respectively, production costs in normal working hours and overtime, inventory-holding costs for both semifinished and finished products, transportation costs between plants, shortage costs, and distribution costs to customers. The second objective (2) seeks to maximise the customer satisfaction level by minimising the amount of products delivered with lateness. Constraints (3) and (4) are the flow balance constraint for the plants in production stages $\mathrm{ST}_{j}, j=1 \ldots N-1$, where production amounts are either stored in the inventory or further transported to its successor plants. Constraints (5) and (6) correspond to the inventory balance in plants from the last production stage. They are equal to the inventory of the previous period and the production amounts minus the shipped products to customers. Constraints (7) and (8) ensure that the quantity shipped from one production plant will be received by its successor. Equation (9) represents that the customer demand over the period must be satisfied or partially backordered. The backorder level of the product for the customer $k$ in the period $t$ is equal to the maximum difference between his/her demand and the quantity shipped from the plants from the last stage to customer zones taking into account distribution lead time. Constraint (10) ensures that the demands of customers for every product under each scenario and for the entire planning horizon are satisfied. In fact, the backorder level at the last period should be zero under the scenario $s$ in order to avoid lost sales. Equation (11) states that the shipping amount of products from the plant for its successor is received in the next period. The limit of backorder's rate is illustrated in constraint (12). Equation (13) limits the production amounts to the random production capacity in regular time under the scenario $s$. The limitation of production capacity in overtime is written in equation (14). Constraint (15) limits the product inventory levels of production plants to their related inventory storage capacities. The shipping quantity of products between plants is delimited in equation (16) and the distribution capacity to customers in constraint (17). The constraint set (18) enforces the nonnegativity of decision variables.

\section{Resolution Approach}

In this paper, we applied one of the efficient multiobjective optimising solvers based on the well-known $\mathcal{E}$-constraint method as it is widely used in the multiobjective productiondistribution planning model.

This approach is firstly defined in [31]. As shown in equations (19) and (20), the biobjective optimisation problem becomes a mono-objective optimisation problem by converting the second objective function into a constraint using the value of $\varepsilon_{2}$.

$$
\begin{gathered}
\min F_{1}, \\
\text { subject to } \quad F_{2} \geq \varepsilon_{2} .
\end{gathered}
$$

The augmented $\varepsilon$-constraint method (AUGMECON) introduced in [32] is employed to generate the Pareto solutions of the multiobjective optimisation problem. The generated Pareto optimal solutions by the $\mathcal{E}$-constraint method may be dominated or inefficient solutions. The augmented $\varepsilon$-constraint technique is suggested to remedy this deficiency. In this method, the inequality constraint (20) of the $\mathcal{E}$-constraint approach is transformed into equality equation (21) by introducing a positive surplus variable $S_{2}$.

$$
\begin{gathered}
\min \quad F_{1}+\gamma * \frac{S_{2}}{r_{2}} \\
F_{2}-S_{2}=\varepsilon_{2}, \\
\text { subject to } S_{2} \varepsilon I R+, \\
S_{2} \varepsilon I R+r_{2}=F_{2}^{\max }-F_{2}^{\min }, \\
\varepsilon_{2}^{k}=F_{2}^{\max }-k * \frac{r_{2}}{q}, \quad k=0,1, \ldots, q,
\end{gathered}
$$

where $r_{2}$ is the range of the second objective function, $\gamma$ is a small positive number generally between $10^{-3}$ and $10^{-6}$, and $S_{2}$ is the introduced surplus variable for the constraint.

To generate the payoff matrix, we should first optimise the two objective functions individually to obtain $F_{1}^{*}$ and $F_{2}^{*}$. Then, the feasible solution range of the considered objective 
functions would be determined with the help of the payoff table and equation (23). Next, we calculate $\varepsilon_{2}^{k}$ by dividing the feasible solution $r_{2}$ into $q$ distributed intervals, as depicted in equation (24). Finally, we optimise the selected objective function with the $\varepsilon$ constraint and then repeat this step considering the different values of $k$ to generate the set of Pareto optimal solutions.

\section{Context of the Industrial Case Study}

6.1. Description of the Case Study. The company considered in this study is called TE INTER, which is an export-oriented company located in Tunisia. The planning concerns a 3month time horizon, with each discrete period of one week. The supply chain network design is composed of 4 production stages, 6 manufacturers, and three customers, as illustrated in Figure 2. The production process includes cutting of the fabric to obtain pattern pieces of the garment, embroidery/serigraphy of these pieces, assembly activity through clothes making process, and finally the packaging operations. TE INTER has the option of subcontracting some orders to local partners for the aim of covering the lack of technical competence and resources (I2) as well as extending the production capacities (I4 and I5).

The finished products are packaged, batched, and distributed to 3 customers located in France. Different transportation modes could be used for delivery operations: trucks are used to tranship the products between plants and the seaport and/or airport for the exportation activities. Each transportation mode is characterized by its own cost, travel time, and capacities. The travel time is considered three periods (three weeks) for a ship and delivered at the same period for the airplane. For the sake of simplicity, we suppose that raw materials (fabrics and accessories) are fully available and with no defects at the beginning of the planning horizon.

Trendy apparel products are characterized by high demand uncertainty, quick response times, and a high risk of obsolescence. Forecasting the demand within the apparel and textile industry is a very difficult issue as demand is often highly volatile and cannot obviously be predicted. These reasons make the decision-makers consider the demand uncertainty in the planning activities. Based on the historical data and experts of the company, we should mention that TE INTER acquires two types of orders from the potential customers: preseason orders and replenishment orders (also called the in-season orders). Preseason orders are that the initial customers' orders comprise the major part of the demand ordered prior to the start of the season. They are firm orders and known with certainty at the beginning of the horizon plan. Accordingly, the due date of the production and the distribution planning of this deterministic demand is 3 months. However, it would be a difficult and hard task to predict the in-season orders because of the emergency of producing and delivering these orders, which serve to prevent shortages resulting from possible initial underestimation of demand or to replace unsold products during the selling season. This is why retailers usually resort to inseason order opportunities after updating their forecasts based on the current sales and trends over the weeks of the current season. The quantity of the in-season orders would be much smaller than that of the preseason orders, and the associated lead time would not exceed few weeks. The second type of uncertainty considered in this industrial field is associated with the internal production capacity. This uncertainty depends on numerous factors and conditions. We should notice that manufacturing processes in the textile and apparel industry are labour-intensive as they are directly connected with the availability of workforce of each production site. In these circumstances, the absenteeism remains the most critical random indicator that would have a huge effect on the actual production planning. Therefore, the actual production time capacity can be obtained by subtracting the absenteeism ratio based on the historical data of one year from total production time resources.

6.2. Data. The considered data correspond to production collection established through 6 months of the summer season.

Overtime production and inventory-holding costs are proportional to regular production cost, following a respective factor of 1.4 and 0.2 . Overtime production capacity is limited to $20 \%$ of the regular production according to the government policy. The distribution costs and capacity depend on the used transportation mode. The preseason demand, which is considered known with certainty at the beginning of the planning horizon, is given in Table 3. In addition, the production capacity without taking into account the absenteeism indicator is reported in Table 4. Based on the historical data of TE INTER, we consider that the customers' replenishment orders or the in-season orders and the production capacity parameters can be either very high, high, medium, or poor, i.e., four scenarios for each random parameter with associated probabilities based on the historical data of the summer season. The probabilities assigned to scenarios represent the importance of each scenario in an uncertain environment. The in-season demand could be estimated as a percentage of the preseason demand. We simply estimate the uncertain rate of workers' availability to define the production capacity by subtracting the ratio of workers' absenteeism and the random threshold of the in-season orders calculated as a percentage of the preseason orders (deterministic demand). The scenarios and the probabilities of the rate of workforce's availability and the ratio of the replenishment orders are depicted in Table 5.

Ratio of workforce's availability for each scenario $=$ (1 - ratio of workforce's absenteeism for each scenario).

$\mathrm{CAPP}_{i t, s}=$ ratio of workforce's availability for each scenario $*$ deterministic production capacity.

$\mathrm{RO}_{p k t, s}=$ ratio of the in-season orders for each scenario $*$ preseason orders.

\section{Computational Results}

This section reports the results of the application of the proposed formulation to the industrial case study as well as 


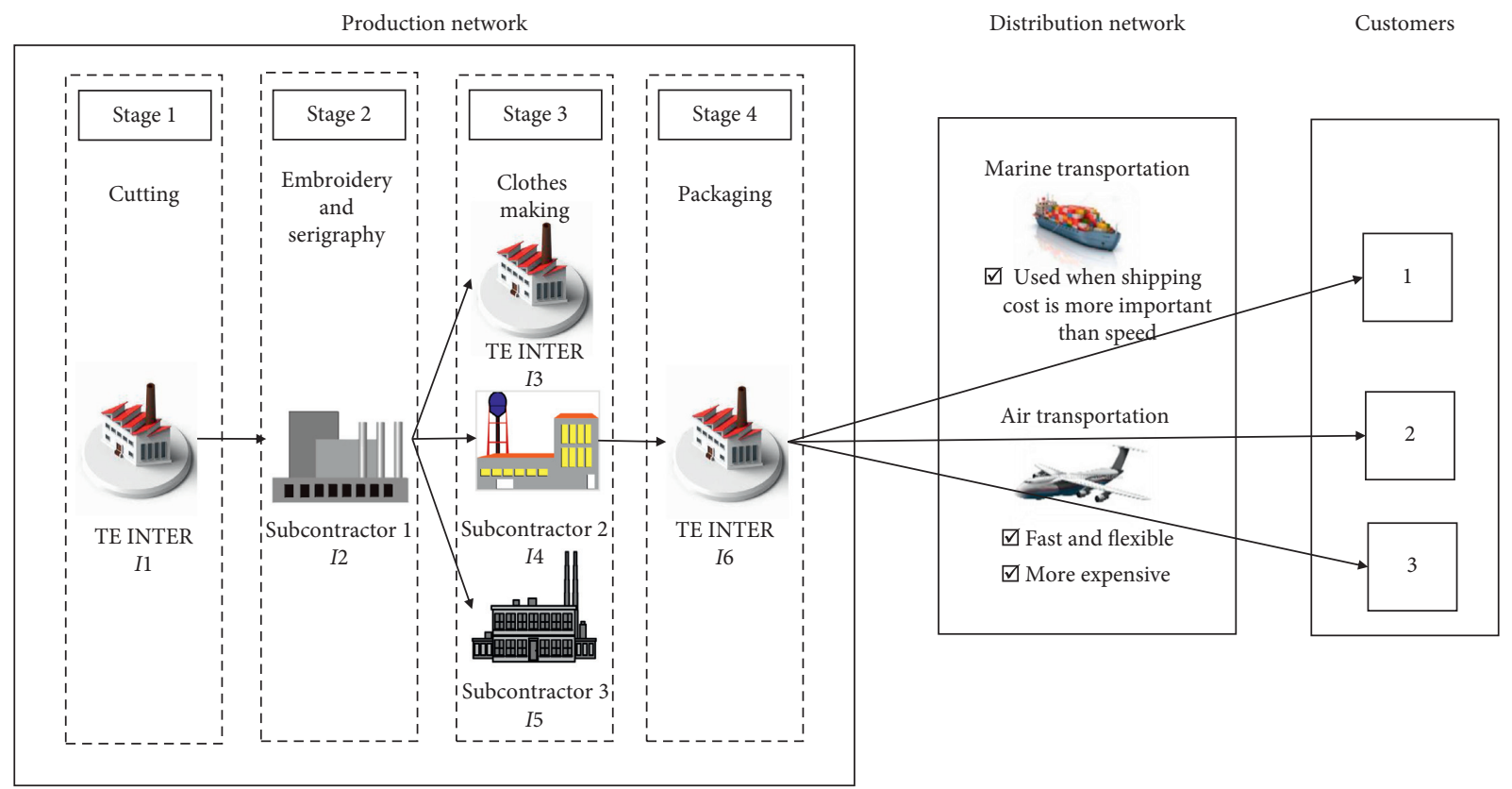

Figure 2: Supply chain design for the case study.

TABLe 3: Deterministic preseason orders (product unit).

\begin{tabular}{|c|c|c|c|c|c|c|}
\hline \multirow{2}{*}{ Product $P$} & \multirow{2}{*}{ Customer $K$} & \multicolumn{5}{|c|}{ Period } \\
\hline & & $T 1-T 8$ & T9 & $T 10$ & $T 11$ & $T 12$ \\
\hline \multirow{3}{*}{$P 1$} & $K 1$ & 0 & 8464 & 0 & 0 & 0 \\
\hline & $K 2$ & 0 & 0 & 8350 & 0 & 0 \\
\hline & K3 & 0 & 0 & 6317 & 7254 & 0 \\
\hline \multirow{3}{*}{$P 2$} & $K 1$ & 0 & 0 & 2766 & 0 & 3620 \\
\hline & $K 2$ & 0 & 5142 & 6930 & 0 & 0 \\
\hline & $K 3$ & 0 & 0 & 0 & 5827 & 0 \\
\hline
\end{tabular}

TABle 4: Deterministic production capacity (minutes).

\begin{tabular}{|c|c|c|c|c|c|c|c|c|c|c|c|c|}
\hline \multirow{2}{*}{ Activities } & \multirow{2}{*}{ Plant $I$} & \multicolumn{11}{|c|}{ Period $T$} \\
\hline & & $T 1$ & $T 2$ & T3 & T4 & T5 & T6 & $T 7$ & $T 8$ & T10 & $T 11$ & T12 \\
\hline Cuttin & $I 1$ & 25410 & 30855 & 29965 & 26995 & 28380 & 30855 & 28875 & 31350 & 27390 & 26895 & 26895 \\
\hline \multirow{2}{*}{ Embroidery/serigraphy } & $I 2$ & 38000 & 32000 & 28500 & 25570 & 31250 & 33500 & 26875 & 32600 & 28450 & 38500 & 33450 \\
\hline & $I 3$ & 94185 & 94185 & 94185 & 95175 & 94185 & 94680 & 94680 & 90825 & 94785 & 96165 & 96165 \\
\hline \multirow[t]{2}{*}{ Clothes making } & $I 4$ & 19950 & 14250 & 14250 & 17100 & 21375 & 25650 & 28500 & 19950 & 14250 & 14250 & 14250 \\
\hline & $I 5$ & 22800 & 25650 & 25650 & 24225 & 15675 & 21375 & 12825 & 14250 & 25650 & 27075 & 27075 \\
\hline Packaging & I6 & 34335 & 40275 & 40275 & 40275 & 39780 & 39780 & 41760 & 47760 & 38775 & 38775 & 38775 \\
\hline
\end{tabular}

TABLE 5: Stochastic parameters for demand and production capacity.

\begin{tabular}{|c|c|c|c|c|c|c|}
\hline \multirow[t]{2}{*}{ Scenario } & \multicolumn{4}{|c|}{ Rate of workforce availability } & \multicolumn{2}{|c|}{$\begin{array}{c}\text { Rate of replenishment } \\
\text { order }\end{array}$} \\
\hline & Cutting activity & Clothes making activity & Packaging activity & Probability & Value & Probability \\
\hline Very high & 0.98 & 0.96 & 0.95 & 0.38 & 0.313 & 0.21 \\
\hline High & 0.88 & 0.9 & 0.832 & 0.27 & 0.204 & 0.31 \\
\hline Medium & 0.8 & 0.84 & 0.765 & 0.19 & 0.136 & 0.36 \\
\hline Low & 0.75 & 0.78 & 0.70 & 0.17 & 0.042 & 0.12 \\
\hline
\end{tabular}


some useful managerial insights that can be highly applicable to the fashion industry.

7.1. Performance of Stochastic Programming Solutions. We use the augmented epsilon constraint (AUGMECON) approach to solve this multiobjective problem. The mathematical model has 1162 continuous decision variables and 822 constraints. The two-stage stochastic programming model and the sampling technique were implemented using LINGO 18 on PC Intel Core i5 with a $2.71 \mathrm{GHz}$ processor and 8 GB memory.

We notice that combining these demand and production capacity uncertainty states resulted in the development of 16 scenarios shown in Table 6.

To assess the importance of the uncertainty effect, we analysed both the EVPI (expected value of perfect information) and the VSS (value of stochastic solution).

The VSS is determined by the difference between the optimal solution of the recourse problem (RP) and the expected result of using the expected value solution (EEV) (equation (25). The latter is obtained by solving the RP with the first-stage decision variables fixed until $\left(t_{1}-1\right)$ at the optimal values achieved by solving the average-scenario deterministic model using the mean values of the uncertain parameters. The VSS is a measure of how much can be saved by implementing the "here and now" solution as opposed to the deterministic expected value solution [33].

$$
\mathrm{VSS}=\mathrm{EEV}-\mathrm{RP} .
$$

We evaluate the EVPI, as shown in equation:

$$
\mathrm{EVPI}=\mathrm{RP}-\mathrm{WS} \text {, }
$$

where RP is the objective value of the two-stage stochastic programming problem and $\mathrm{WS}=\delta(s) * \mathrm{WS}(s)$ is the expected wait-and-see solution, which expresses the objective value of the deterministic problem for the scenario $s$.

Table 7 depicts the performance of stochastic programming solutions considering the implementation of the problem of the two-stage stochastic programming approach considering both random replenishment orders and production capacity. For each value of epsilon, we obtain relatively high values of the EVPI and VSS, which imply that it is important to consider uncertainty. The EVPI ranges from 24666.98 to 30861.44 , which is approximately $6.691 \%$ to $8.091 \%$ of the two-stage stochastic objective value (RP), and The VSS ranges from 20851.97 to 35982.28 , which is approximately $5.62 \%$ to $9.11 \%$ of the RP. Based on equations (25) and (26), diverse conclusions can be withdrawn by inspecting Table 8 . In fact, the great values of the EVPI mean that incomplete information about the future may prove costly. Consequently, neglecting uncertainty in these cases deteriorates significantly the demand fulfillment policies and the capacity planning strategies. Besides, it is clear from the interpretation of the VSS that the solutions of the proposed two-stage stochastic programming model exceed those of the deterministic model. Accordingly, we point out that ignoring uncertainty via replacing the random parameters
TABle 6: Definition of stochastic scenarios.

\begin{tabular}{lccc}
\hline Scenario & $\begin{array}{c}\text { Replenishment } \\
\text { order uncertainty }\end{array}$ & $\begin{array}{c}\text { Production } \\
\text { capacity } \\
\text { uncertainty }\end{array}$ & $\begin{array}{c}\text { Probability } \delta \\
(s)\end{array}$ \\
\hline S1 & Very high & Very high & 0.0798 \\
S2 & Very high & High & 0.1178 \\
S3 & Very high & Medium & 0.1368 \\
$S 4$ & Very high & Low & 0.0456 \\
S5 & High & Very high & 0.0567 \\
S6 & High & High & 0.037 \\
$S 7$ & High & Medium & 0.0972 \\
$S 8$ & High & Low & 0.0324 \\
$S 9$ & Medium & Very high & 0.0399 \\
$S 10$ & Medium & High & 0.0589 \\
$S 11$ & Medium & Medium & 0.0684 \\
$S 12$ & Medium & Low & 0.0228 \\
$S 13$ & Low & Very high & 0.0336 \\
$S 14$ & Low & High & 0.0496 \\
$S 15$ & Low & Medium & 0.0576 \\
$S 16$ & Low & Low & 0.0192 \\
\hline
\end{tabular}

by their mean value leads to nonoptimised planning during making decisions. Furthermore, VSS/RP (\%) has its highest value in case of the total customer satisfaction level $(F 2=1)$ which proves that considering uncertain factors has become more critical. These results imply the potential worth of obtaining more accurate forecast as well as efficient stochastic solutions.

Figure 3 presents the Pareto optimal solution of the RP and EEV and the solution provided by TE INTER. It clearly indicates that the considered objective functions are conflicting. It seems that an increase in one causes a decline in the other one because the on-time delivery loading effect will entail a strong percentage augmentation of distribution costs and overtime production costs, which strongly leads to decreasing profit.

The total cost of the company's current practice is 421247.31 (monetary unit), and the customer satisfaction level is $56 \%$. Our numerical results show that our stochastic approach not only provides better stochastic solutions (RP) than the expected value solution (EEV) but also is better than those proposed by the company's planner, as clearly depicted in Figure 3. In fact, we notice that the improvements stated between stochastic solutions (RP) and solution provided by the company are evaluated as $12.48 \%$ in terms of total costs and $28.77 \%$ in terms of the service level. To enhance the practitioners' understanding of these gains, it is important to consider the integration of production and distribution planning when demand and production capacity parameters are uncertain. Hence, the proposed model uses historical data and mathematical model that hold the ability to manage uncertain information. Anticipating absenteeism and demand helps firms achieve their targets, customer satisfaction, and increase productivity as well as flexibility and responsiveness of the supply chain.

Figure 4 gives information about the total costs, regular production costs, overtime production costs, backorder costs, and transportation costs of each mode for the different scenarios $(\varepsilon=0.72)$. 
TABLe 7: Probability assumptions in various scenarios.

\begin{tabular}{lccccccccc}
\hline \multirow{2}{*}{ Scenario } & \multicolumn{3}{c}{ Variation of in-season demand's probabilities } & \multicolumn{3}{c}{ Variation of production capacity's probabilities } \\
& Case study & Instance 1 & Instance 2 & Instance 3 & Instance 4 & Case study & Instance 5 & Instance 6 & Instance 7 \\
\hline 1 & 0.38 & 0.20 & 0.20 & 0.20 & 0.25 & 0.21 & 0.40 & 0.20 & 0.20 \\
2 & 0.27 & 0.40 & 0.20 & 0.20 & 0.25 & 0.31 & 0.20 & 0.40 \\
3 & 0.19 & 0.20 & 0.40 & 0.20 & 0.25 & 0.36 & 0.20 & 0.20 & 0.20 \\
4 & 0.16 & 0.20 & 0.20 & 0.40 & 0.25 & 0.12 & 0.20 & 0.20 & 0.40 \\
\hline
\end{tabular}

TABLE 8: Performance of stochastic programming solutions considering the uncertainties of replenishment orders and production capacity.

\begin{tabular}{|c|c|c|c|c|c|c|c|c|c|c|}
\hline $\begin{array}{l}\text { Pareto } \\
\text { solutions }\end{array}$ & $\varepsilon(k)$ & $\begin{array}{l}\mathrm{CPU} \\
(\mathrm{s})\end{array}$ & $F 2$ & $\begin{array}{c}\mathrm{RP} \\
\text { (monetary } \\
\text { unit) }\end{array}$ & $\begin{array}{c}\text { WS } \\
\text { (monetary } \\
\text { unit) }\end{array}$ & $\begin{array}{c}\text { EVPI } \\
\text { (monetary unit) }\end{array}$ & $\begin{array}{l}\text { EVPI/RP } \\
(\%)\end{array}$ & $\begin{array}{c}\text { EEV } \\
\text { (monetary } \\
\text { unit) }\end{array}$ & $\begin{array}{c}\text { VSS } \\
\text { (monetary } \\
\text { unit) }\end{array}$ & $\begin{array}{c}\text { VSS/RP } \\
(\%)\end{array}$ \\
\hline Solution 1 & 0.72 & 5 & 0.786 & 368661.1 & 343994.1 & 24666.98 & 6.691 & 391913 & 23251.98 & 6.31 \\
\hline Solution 2 & 0.74 & 3.97 & 0.795 & 369187.1 & 344018.6 & 25168.45 & 6.817 & 391913 & 22725.95 & 6.16 \\
\hline Solution 3 & 0.77 & 3.78 & 0.803 & 370035.5 & 344072.7 & 25962.76 & 7.016 & 391913 & 21877.56 & 5.91 \\
\hline Solution 4 & 0.80 & 4.11 & 0.817 & 371061.1 & 344167.5 & 26893.61 & 7.248 & 391913 & 20851.97 & 5.62 \\
\hline Solution 5 & 0.83 & 4.2 & 0.841 & 372422.1 & 344326.2 & 28095.87 & 7.544 & 393428.6 & 21006.51 & 5.64 \\
\hline Solution 6 & 0.86 & 3.8 & 0.861 & 374213.1 & 344845.8 & 29367.34 & 7.848 & 396477.7 & 22264.55 & 5.95 \\
\hline Solution 7 & 0.89 & 9.16 & 0.890 & 376507.8 & 346177.6 & 30330.18 & 8.056 & 399137.2 & 22629.45 & 6.01 \\
\hline Solution 8 & 0.91 & 10.38 & 0.910 & 378317.7 & 347706.2 & 30611.49 & 8.091 & 400986.6 & 22668.95 & 5.99 \\
\hline Solution 9 & 0.94 & 4.09 & 0.940 & 381852.8 & 350953.5 & 30899.3 & 8.092 & 405442.2 & 23589.34 & 6.18 \\
\hline Solution 10 & 0.97 & 4.99 & 0.970 & 387226.8 & 356685.5 & 30541.21 & 7.887 & 413829.9 & 26603.14 & 6.87 \\
\hline Solution 11 & 1.00 & 3.47 & 1.000 & 394931.5 & 364070.1 & 30861.44 & 7.814 & 430913.8 & 35982.28 & 9.11 \\
\hline
\end{tabular}

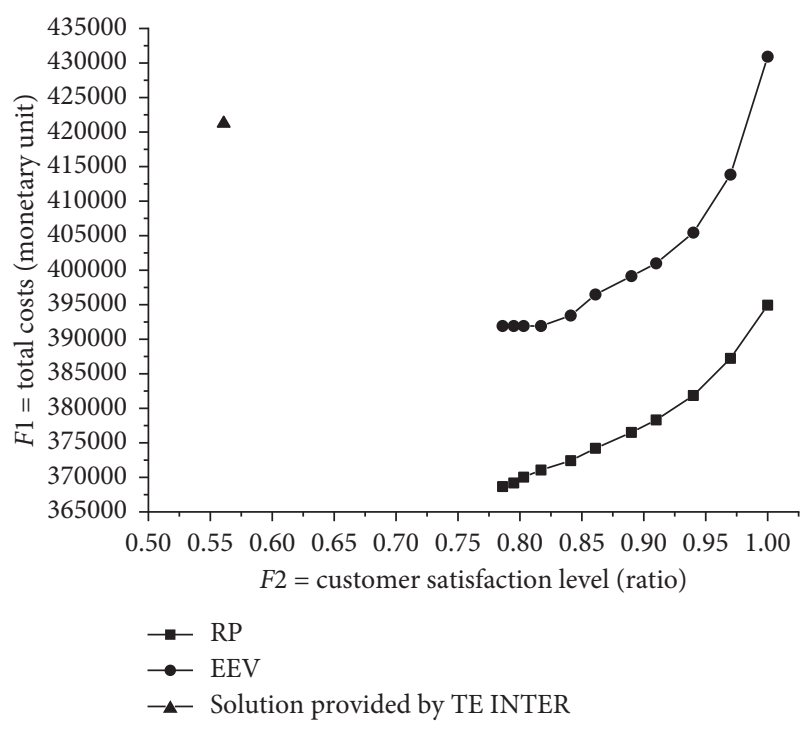

Figure 3: Pareto set of RP and EEV solutions and the solution provided by TE INTER.

Based on the numerical results in Figure 4, we note that the total costs $(F 1)$ increase dramatically, while the customers' orders increase or the production capacity decreases. In fact, fixing, for example, of all "very high" in-season order scenarios $(S 1, S 2, S 3$, and $S 4)$, we conclude that $F 1$ increases when the production capacity is becoming lower. Taking into account the same production capacity scenario (for example, for medium scenario of production capacity: $S 3$, $S 7, S 11$, and $S 15)$, it is obvious that overall costs (F1) increase in case of the increasing replenishment orders. The results reveal that the main contributions of the cost increasing in case of increasing quantity of the random orders urge the

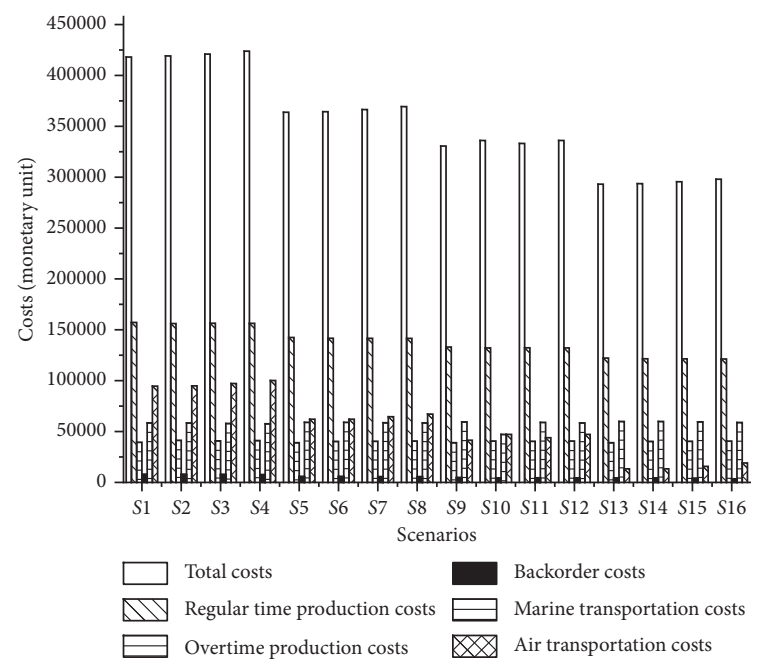

Figure 4: Distribution of costs $(\varepsilon=0.72)$.

decision maker to react and plan more overtime production and use the airplane as a tool to speed up the production and the distribution activities. In conclusion, in order to hedge the huge effect of uncertainties, the practitioner would choose to procure either more overtime production or delivery planning using the combined sea-air transportation to fulfill the order delivery on time.

7.2. Sensitivity Analysis. Here, we focus on a single-factor sensitivity analysis to study how the variation of the scenario probabilities of uncertain parameters affects the performance of the model.

We generate randomly 7 instances conserving the same supply chain structure of the industrial case study as we can 
see in Table 7. We firstly vary in instances 1-4 the probabilities of the in-season demand and consider the same probability of the production capacity of the case study. As for instance 5-6, we assume the same in-season demand probability and we consider a variation in production capacity's probability. In each instance, a scenario is considered to be far more likely than other economic scenarios.

As shown in Figure 5, the total cost is more sensitive to the probability of uncertain demand compared with the probability of production capacity for instances from 1 to 6 . This observation could be explained by the impact of demand on the supply chain network performance. However, the cost augments slightly with the distribution of production capacity in these numerical examples. In instance 7, the cost increases dramatically assuming that the $4^{\text {th }}$ scenario of lowest production capacity has the highest probability. This might be explained by planning more overtime production and air transportation.

7.3. Managerial Insights. Demand uncertainty is the major challenge for any organisation in the current competitive world. In our case study, a part of the demand known as the in-season order is stochastic. In the real practice of the company, managers receive these unexpected orders during the selling season of the garments. The uncertain amount of these orders should be produced and delivered within a shorter time lapse to satisfy the customers and end users. On the other side, employee absenteeism is also identified as a crucial random factor that affects labour productivity and the quick response of the orders. Generally, the term "absenteeism" refers to unscheduled and unapproved absences of workers from the workplace. Based on our empirical findings, we provide decisionmakers with a pathway to follow for enhancing the level of the planning. The improvement area is achieved by a strong trade-off of the production in normal working hours and overtime, subcontracting, combined sea-air distribution, and backordering costs, whereas the current strategy based on the sequential planning method focuses solely on production. In terms of the customer service level, distribution network restructuring provides greater benefit to the apparel industry. In fact, the proposed model takes advantage of the airway transportation option to save time on trips, and this consequently increases on-time deliveries. To solve the problem of the absenteeism fluctuation, decision-makers can boost productivity by planning the annual leave taking into account the low and peak production season. Thus, the managers would consider changing schedules when appropriate to accommodate different employee needs since the planning becomes more viable especially in case of high demand and low productivity. Therefore, it is believed that these findings can guide decision-makers in making improvements with regard to costs and service level performance. This paper not only provides insights for policy-makers in terms of policy support but also contributes to the literature particularly within a competitive environment such as the textile-apparel industry.

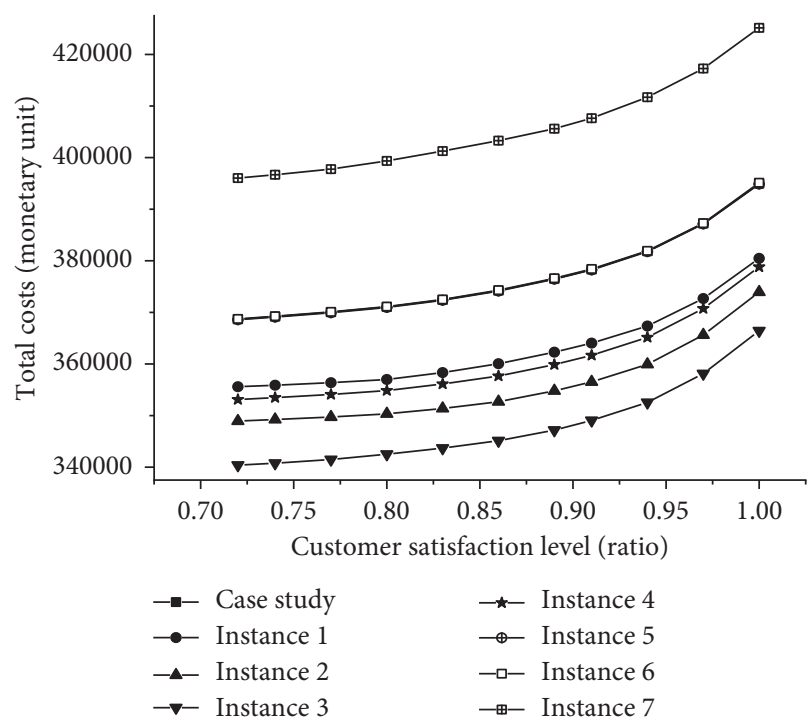

FIgURE 5: Pareto optimal solutions for the different considered instances.

\section{Conclusion and Perspectives}

This paper presents a new formulation of an integrated production-distribution planning problem in a stochastic multisite, multiproduct, multiperiod, and intermodal supply chain context. Two objectives are considered: minimising the total costs and maximising the customer satisfaction level in terms of orders delivered on time. A two-stage stochastic programming approach is developed to deal with demand and production capacity uncertainties. Results not only provide optimal production and distribution planning but also show the importance of considering uncertainties in the stochastic environment. A case study inspired from the manufacturing company producing trading clothes has been managed to illustrate and validate the stochastic model. Significant values of EVPI and VSS are obtained showing, respectively, the expected gain of perfect information and the outperformance of the stochastic solutions (RP) over the expected value solution (EEV). These results justify the effectiveness of the proposed two-stage programming approach. Compared to real practice of the company, the observed improvements are evaluated as $12.48 \%$ in total costs and $28.77 \%$ in the customer service level. It is worth mentioning that the application of the proposed model may give some interesting managerial insights for decisionmakers regarding managing the fluctuation of the uncertainties of the apparel supply chain network. For instance, managers can settle a planning of annual leaves taking into account the peak and low production season in order to foster the production capacity and the flexibility of their plants.

For the future work, other sources of randomness affecting the manufacturing systems such as quality and supply uncertainties can be integrated. Another interesting issue consists of developing a risk management approach to ensure the robustness of the stochastic model. Finally, solving substantially larger production-distribution planning problems, 
compared to those we addressed in our real case study, may call for the development of metaheuristics that can reach optimal solutions within reasonable lengths of time.

\section{Data Availability}

The data provided by the company TE INTER used to support the findings of this study are included within the article.

\section{Conflicts of Interest}

The authors declare that they have no conflicts of interest.

\section{Acknowledgments}

The authors gratefully acknowledge TE INTER's staff for their serious cooperation making this research possible and providing the industrial valorisation of the developed research. The authors also would like to thank LINDO Systems for providing a free educational research license of the extended version of the LINGO 17.0 software package.

\section{References}

[1] D. Peidro, J. Mula, R. Poler, and F.-C. Lario, "Quantitative models for supply chain planning under uncertainty: a review," The International Journal of Advanced Manufacturing Technology, vol. 43, no. 3-4, pp. 400-420, 2009.

[2] M. Fisher and A. Raman, "Reducing the cost of demand uncertainty through accurate response to early sales," Operations Research, vol. 44, no. 1, pp. 87-99, 1996.

[3] Y. B. Park, "An integrated approach for production and distribution planning in supply chain management," International Journal of Production Research, vol. 43, no. 6, pp. 1205-1224, 2005.

[4] Z.-L. Chen, "Integrated production and outbound distribution scheduling: review and extensions," Operations Research, vol. 58, no. 1, pp. 130-148, 2010.

[5] B. Fahimnia, R. Z. Farahani, R. Marian, and L. Luong, "A review and critique on integrated production-distribution planning models and techniques," Journal of Manufacturing Systems, vol. 32, no. 1, pp. 1-19, 2013.

[6] A. H. Niknamfar, S. T. A. Niaki, and S. H. R. Pasandideh, "Robust optimization approach for an aggregate productiondistribution planning in a three-level supply chain," The International Journal of Advanced Manufacturing Technology, vol. 76, no. 1-4, pp. 623-634, 2015.

[7] K. Govindan and M. Fattahi, "Investigating risk and robustness measures for supply chain network design under demand uncertainty: a case study of glass supply chain," International Journal of Production Economics, vol. 183, pp. 680-699, 2015.

[8] Y. Feng, A. Martel, S. D’Amours, and R. Beauregard, "Coordinated contract decisions in a make-to-order manufacturing supply chain: a stochastic programming approach," Production and Operations Management, vol. 22, no. 3, pp. 642-660, 2013.

[9] H. Badri, S. M. T. Fatemi Ghomi, and T.-H. Hejazi, "A twostage stochastic programming approach for value-based closed-loop supply chain network design," Transportation Research Part E: Logistics and Transportation Review, vol. 105, pp. 1-17, 2017.
[10] S. A. Torabi and E. Hassini, "Multi-site production planning integrating procurement and distribution plans in multiechelon supply chains: an interactive fuzzy goal programming approach," International Journal of Production Research, vol. 47, no. 19, pp. 5475-5499, 2009.

[11] G. K. Badhotiya, G. Soni, and M. L. Mittal, "Fuzzy multiobjective optimization for multi-site integrated production and distribution planning in two echelon supply chain," The International Journal of Advanced Manufacturing Technology, vol. 102, no. 1-4, pp. 635-645, 2019.

[12] A. Ait-Alla, M. Teucke, M. Lütjen, S. Beheshti-kashi, and H. Reza Karimi, "Robust production planning in fashion apparel industry under demand uncertainty via conditional value at risk," Mathematical Problems in Engineering, vol. 2014, Article ID 901861, 10 pages, 2014.

[13] A. Jabbarzadeh, M. Haughton, and F. Pourmehdi, "A robust optimization model for efficient and green supply chain planning with postponement strategy," International Journal of Production Economics, vol. 59, pp. 1-58, 2018.

[14] F. Goodarzian and H. Hosseini-Nasab, "Applying a fuzzy multi-objective model for a production-distribution network design problem by using a novel self-adoptive evolutionary algorithm," International Journal of Systems Science: Operations \& Logistics, pp. 1-23, 2019.

[15] K. Govindan, M. Fattahi, and E. Keyvanshokooh, "Supply chain network design under uncertainty: a comprehensive review and future research directions," European Journal of Operational Research, vol. 263, no. 1, pp. 108-141, 2017.

[16] J. Coenen, E. Rob, C. M. Van Der Heijden, and A. C. R. Van Riel, "Understanding approaches to complexity and uncertainty in closed-loop supply chain management: past findings and future directions," Journal of Cleaner Production, vol. 40, 2018.

[17] A. Gupta and C. D. Maranas, "Managing demand uncertainty in supply chain planning," Computers \& Chemical Engineering, vol. 27, no. 8-9, pp. 1219-1227, 2003.

[18] A. Entezaminia, M. Heidari, and D. Rahmani, "Robust aggregate production planning in a green supply chain under uncertainty considering reverse logistics : a case study," The International Journal of Advanced Manufacturing Technology, vol. 90, no. 5-8, pp. 1507-1528, 2017.

[19] G. B. Dantzig and B. George, "Linear programming under uncertainty," Management Science, vol. 1, no. 3-4, pp. 197206, 1955.

[20] I. Safra and A. Jebali, "Capacity planning in textile and apparel supply chains," IMA Journal of Management Mathematics, vol. 30, no. 2, pp. 209-233, 2018.

[21] Z. He, Z. Guo, and J. Wang, "Integrated scheduling of production and distribution operations in a global MTO supply chain," Enterprise Information Systems, vol. 13, no. 4, pp. $490-514,2018$.

[22] C. Weskamp, A. Koberstein, F. Schwartz, L. Suhl, and S. Voß, "A two-stage stochastic programming approach for identifying optimal postponement strategies in supply chains with uncertain demand," Omega, vol. 83, 2018.

[23] H. Mohammadi and R. Mohd, "Integrated supply chain planning under uncertainty using an improved stochastic approach," Applied Mathematical Modelling, vol. 35, no. 6, pp. 2618-2630, 2011.

[24] S. M. J. Mirzapour Al-e-hashem, A. Baboli, S. J. Sadjadi, and M. B. Aryanezhad, "A multiobjective stochastic productiondistribution planning problem in an uncertain environment considering risk and workers productivity," Mathematical 
Problems in Engineering, vol. 2011, Article ID 406398, 14 pages, 2011.

[25] G. Guillén, F. D. Mele, M. J. Bagajewicz, A. Espuña, and L. Puigjaner, "Multiobjective supply chain design under uncertainty," Chemical Engineering Science, vol. 60, no. 6, pp. 1535-1553, 2005.

[26] A. Azaron, K. N. Brown, S. A. Tarim, and M. Modarres, "A multi-objective stochastic programming approach for supply chain design considering risk," International Journal of Production Economics, vol. 116, no. 1, pp. 129-138, 2008.

[27] M. Rahimi, V. Ghezavati, and F. Asadi, "A stochastic riskaverse sustainable supply chain network design problem with quantity discount considering multiple sources of uncertainty," Computers \& Industrial Engineering, vol. 130, pp. 430-449, 2019.

[28] S. H. R. Pasandideh, S. T. A. Niaki, K. Asadi, and K. Asadi, "Biobjective optimization of a multi-product multi-period threeechelon supply chain problem under uncertain environments: NSGA-II and NRGA," Information Sciences, vol. 292, pp. 57-74, 2015.

[29] Y. Nemati and M. H. Alavidoost, "A fuzzy bi-objective MILP approach to integrate sales, production, distribution and procurement planning in a FMCG supply chain," Soft Computing, vol. 23, no. 13, pp. 4871-4890, 2019.

[30] D. Walkup and R. J.-B. Wets, "Stochastic programs with recourse," SIAM Journal on Applied Mathematics, vol. 15, no. 5, pp. 1299-1314, 1967.

[31] Y. Y. Haimes, L. S. Lasdon, and D. A. Wismer, "On a bicriterion formulation of the problems of integrated system identification and system optimization," IEEE Journals \& Magazines, vol. 47, pp. 296-297, 1971.

[32] G. Mavrotas, "Effective implementation of the $\varepsilon$-constraint method in multi-objective mathematical programming problems," Applied Mathematics and Computation, vol. 213, no. 2, pp. 455-465, 2009.

[33] J. R. Birge, "Models and model value in stochastic programming," Annals of Operations Research, vol. 59, no. 1, pp. 1-18, 1995. 